Techniques \& Culture

\title{
L'introduction tardive du diable et de la brouette au Moyen-Orient
}

Un problème pour l'anthropologie des diffusions

Didier Gazagnadou

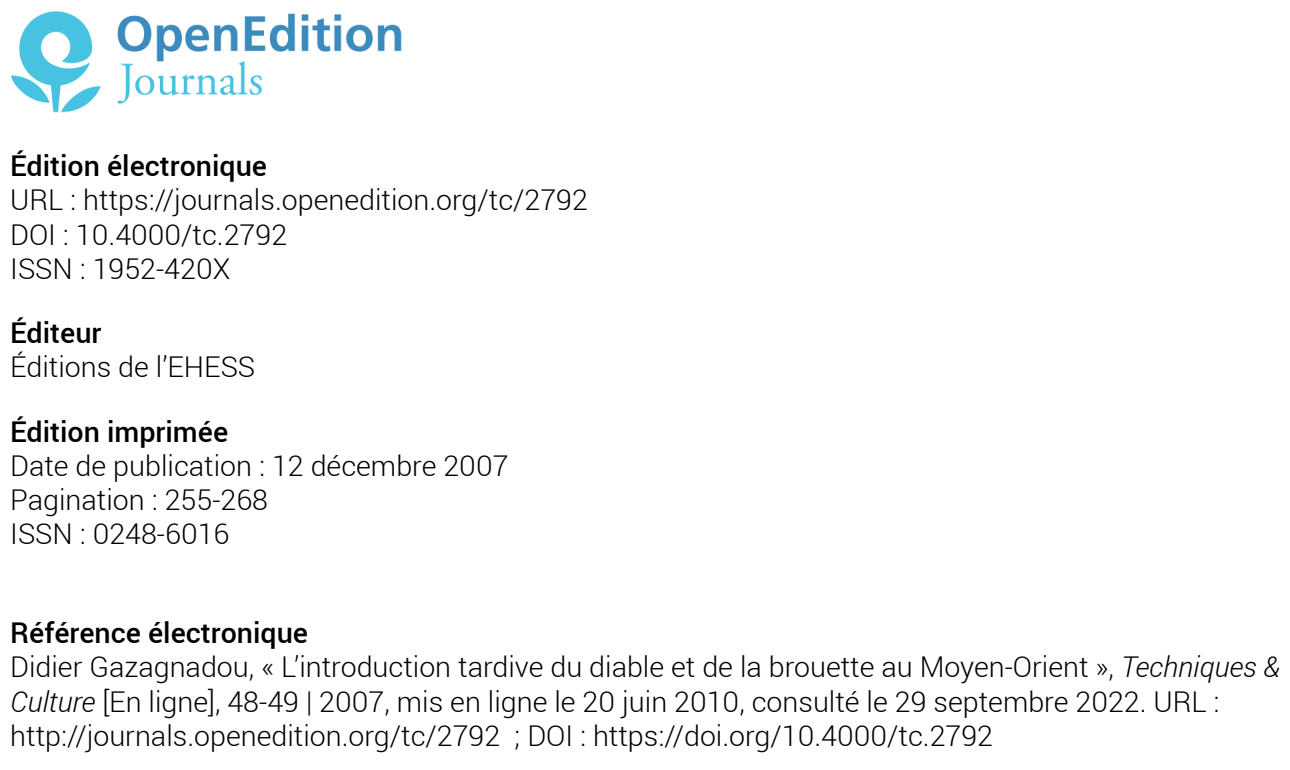

Tous droits réservés 


\section{Didier Gazagnadou*}

\section{L'introduction tardive du diable et de la brouette au Moyen-Orient Un problème pour l'anthropologie des diffusions}

La prévalence du transport par animaux de bât au Moyen-Orient au détriment de lutilisation des véhicules à roues est une question technique et politique encore problématique tout comme reste posé le problème de la diffusion de ces véhicules à roues. Cette note soulève de nouveau ces questions en exposant le cas de deux petits véhicules, la brouette et le diable, du fait de leur introduction tardive en Égypte et en Iran.

Brouette, diable, roue, diffusion technique, Chine, Iran, Égypte.

Les techniques et les moyens de transport sont, pour l'anthropologie et l'histoire des techniques, des problèmes importants. Les véhicules à roues, dont le rôle fut essentiel, restent malheureusement peu étudiés comme du reste les techniques en général ${ }^{1}$, et en particulier dans le monde iranien et la civilisation islamique.

Le système technique de transport en Iran et en Égypte possède une des caractéristiques essentielles de celui des sociétés du Moyen-Orient: la marginalisation (du $\mathrm{III}^{\mathrm{e}}$ siècle ap. J.-C. environ jusquau milieu du $\mathrm{XX}^{\mathrm{e}}$ siècle) du transport par roulage (véhicules à roues à traction animale) au

\footnotetext{
* Professeur d'anthropologie, Université Paris 8, département de sociologie (UFR 4) \& équipe "Anthropologie Comparative des Sociétés Musulmanes" (axe sociologie comparative), Laboratoire d'anthropologie sociale, Collège de France-CNRS-EHESS.gazad@wanadoo.fr

${ }^{1}$ En ce qui concerne l'anthropologie, Robert Cresswell, dans son dernier ouvrage (1996: 330), pose la question plus clairement : «Ce serait à nous, $[. .$.$] de nous demander pourquoi nous avons laissé stagner la$ technologie pendant des années ».
}

Techniques \& culture 48-49, 2007: 255-268 
profit du transport par animaux de bât (mulets, chevaux, ânes, dromadaires, chameaux, bovins) et par portage humain. Bien que nous ayons déjà discuté (Gazagnadou 1999) et montré notre désaccord partiel avec la thèse de R.W. Bulliet (I990) sur les causes de cette prévalence du portage par animaux de bât dans l'aire moyen-orientale puis islamique, elle est un fait incontestable, quasiment jusquau milieu du siècle dernier. Nous examinerons de nouveau cette question à la lumière de cas concrets qui confirment cette marginalisation des véhicules à roues, en prenant l'exemple de la brouette puis plus brièvement celui du diable et de leur introduction en Iran et en Égypte.

\section{La brouette}

Bien que ce petit véhicule de transport à roue soit aujourd'hui connu dans le monde entier, son histoire est encore obscure et na que très peu retenu l'attention des technologues malgré sa grande utilité dans de très nombreuses activités sociales et économiques.

Au milieu du XVIII ${ }^{\mathrm{e}}$ siècle, l'Encyclopédie de Diderot et D’Alembert ( $175 \mathrm{I})$ donnait de la brouette la définition suivante :

«Brouette : Petite machine faite en forme de charrette, qui n'a qu'une roue, et que celui qui s'en sert pousse devant soi par le moyen de deux espèces de timons, soutenus d'un côté par l'essieu de la roue, et de l'autre par les mains de celui qui machine qui pour cet effet se met au milieu. "

Cette description est tardive, on trouve en effet des représentations de la brouette en Europe dès le milieu du XIII ${ }^{\mathrm{e}}$ siècle (Gille I983: 82). Ce petit véhicule permet le transport, par un seul homme, de charges d'environ Ioo kg. Pratique, mobile, individuel, il permet le transport sur des voies et des passages étroits, dans les chantiers ainsi que dans les ruelles des halles et des villes (médiévales et modernes), les chemins de campagnes et dans les fermes. De nos jours, on le retrouve dans à peu près tous les endroits du monde où se pratiquent des activités commerciales, agricoles et de construction. Quant à son utilité, rappelons ce qu'écrivait à juste titre Bertrand Gille (1983: 79):

"Qu’on ne dise pas qu’il s'agit là d’un instrument secondaire. Elle a largement facilité, et très largement, l'agriculture et les grands travaux d'utilité publique. Bien plus, elle est restée, jusqu'à nos jours, beaucoup plus que d'autres, un instrument indispensable que l'on voit dans toutes les campagnes, que l'on rencontre dans tous les chantiers. " 
On ajoutera qu'avec le développement de l'habitat pavillonnaire et du jardinage de loisir, la brouette est présente dans de nombreux foyers.

De la brouette, on peut dire qu'elle est d'une part « une civière dont l'un des porteurs a été remplacé par une ou deux roues " (Camasara 1925: $2^{\text {ème }}$ partie) et d'autre part, le développement individualisé de la charrette, par miniaturisation; ce qui renvoie à lidée d'Haudricourt, pour qui la brouette est un char portatif ${ }^{2}$. C'est aussi la substitution de l'énergie humaine à l'énergie animale, répondant à un souci d'efficacité et de maniabilité pour des tâches destinées à un seul homme. Il s'agit enfin de la substitution d'un mode de portage mécanique au seul portage à dos d'homme ou animal : la brouette est une prothèse technologique. Bien quil y ait quelques exemples en Europe ou en Chine de brouettes tirées par l'homme, d'une manière générale, on pousse une brouette, sauf lorsqu’elle est vide.

Sur l'histoire de la brouette

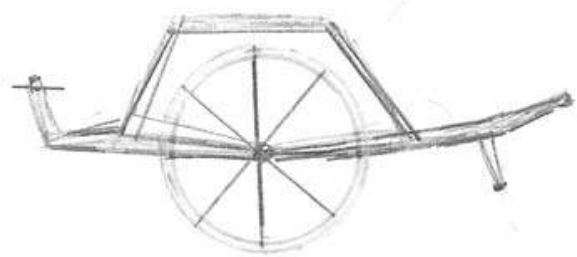

Figure 1. Brouette de type chinois.

En Chine, la brouette à une roue centrale (fig. I), parfois munie d'une voile, est attestée 3 entre le $\mathrm{I}^{\text {er }}$ siècle et le III $^{\mathrm{e}}$ siècle (Gernet 2000 : I28; Needham

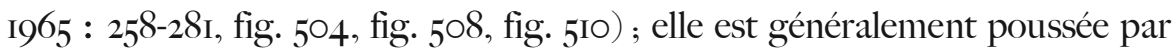
un homme et son nom en chinois le confirme: du lun shou tui che

\footnotetext{
2 Entretien privé avec André-Georges Haudricourt de janvier 1996.

3 Cependant, il existe également en Chine des exemples de brouettes à une ou deux roues situées devant le caisson, mais nous n'en connaissons pas les dates des toutes premières attestations.
} 
("véhicule à une roue poussé à l’aide des mains "⿱乛龰). Quelquefois, des iconographies nous montrent un second homme, un âne ou un mulet attaché par une corde à l'avant de la brouette et qui aide à la traction.

La brouette apparaît dans liconographie de l'Europe occidentale au cours du XIII ${ }^{\mathrm{e}}$ siècle (cf. le manuscrit I77 de Dublin datant de I24O-I25O, Lewis 1994). Précisons toutefois que c'est surtout dans l'iconographie médiévale du XIV ${ }^{\mathrm{e}}$ siècle qu'on la retrouve (Gille 1994). L'étymologie du mot brouette, pour A.-G. Haudricourt comme pour B. Gille, nous conduit à l'ancien français béroué, lui-même dérivé du bas latin birota, ce qui suppose bien deux roues à l’origine. Or, les représentations médiévales que reproduisent B. Gille et P. Mane'5 nous montrent beaucoup plus de brouettes à une seule roue qu'à deux roues. La brouette à une roue devant le caisson est le modèle dominant (fig. 2) en Europe et a entraîné la disparition de celle à deux roues, probablement du fait que, si cette dernière a l'avantage d'être plus stable dans le roulage, elle s'avère plus difficile à faire virer. D'une manière générale, la brouette à deux roues, surtout chargée, est plus difficile à manœuvrer et à vider qu'une brouette à une roue. Le «triomphe» de la brouette à une roue a une origine technique : plus d'aisance et d'efficacité dans l'utilisation et pénibilité moindre pour l'ouvrier.

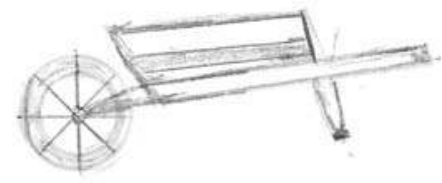

Figure 2. Brouette de type européen.

\footnotetext{
${ }^{4}$ Je remercie Georges Métailié (CNRS, Centre A. Koyré) pour ses traductions du chinois et ses remarques sur l'ensemble du texte.

${ }^{5}$ Je remercie Perrine Mane (CNRS) pour les informations qu'elle a bien voulu me communiquer, notamment une liste de brouettes médiévales où l'on trouve un nombre non négligeable de brouettes à deux roues.
} 
Ce petit véhicule sinscrit manifestement dans un système technique où les véhicules à roues jouent un rôle important dans les transports: un système dans lequel lutilisation de la charrette et/ou du chariot était relativement courante. Cétait le cas de la Chine pour ce qui concerne le char ou la charrette au moins depuis l'époque du premier empereur Qin Shi Huangdi (22I-2IO av. J.-C.).

La brouette sinsère dans la structure technique d'organisation du travail d'une société sédentaire et agricole où les paysans et les artisans empruntent des chemins et des voies de passage étroits, sur lesquels le roulage est possible.

\section{L'introduction de la brouette en Égypte, en Iran et dans la civilisation islamique}

Lintroduction et la découverte de la brouette dans le monde arabe semblent bien remonter à la toute fin du XVIII ${ }^{\mathrm{e}}$ siècle, et en Égypte plus particulièrement au moment de l'expédition française, dont l'écrivain égyptien Abd al-Rahmân al-Jabarti a été le témoin et l'observateur ; il écrit à ce sujet :

«Ils [les Français] recouraient à des instruments faciles à manier et épargnant la peine, ce qui permettait une exécution rapide des travaux. Ainsi, au lieu de paniers ou de récipients, ils utilisaient de petites charrettes $\left[\right.$ brouettes ${ }^{6}$ qui avaient deux bras allongés par-derrière; on les remplissait de terre, d'argile ou de pierres par-devant avec grande facilité, l'équivalent de cinq paniers; ensuite on prenait en main $^{7}$ les deux bras [de la brouette], on poussait devant soi et la charrette roulait sur sa roue avec moindre peine jusqu'au chantier ; on les vidait enfin, en la penchant d'une main, sans aucune fatigue " (Jabarti I979: 89).

La description de Jabarti est intéressante, d'une part parce qu'elle confirme qu'il s’agissait d'un instrument inconnu à la fin du $\mathrm{XIX}^{\mathrm{e}}$ siècle en Égypte, et d'autre part, d'un point de vue techno-économique, elle montre bien lintérêt de la brouette. Il convient toutefois d'ajouter que la brouette n'a pas été utilisée systématiquement en Égypte avant le $\mathrm{XX}^{\mathrm{e}}$ siècle et que, jusquà cette époque, on a continué à transporter à dos d’homme et

\footnotetext{
${ }^{6}$ L'expression arabe exacte employée par Jabarti est : 'arabât sarîrat; la traduction de Cuoq par petites charrettes n'est pas rigoureuse, car le mot 'arabâ désigne un char, une charrette ou un chariot, c'est-à-dire des véhicules à deux ou quatre roues, donc 'arabat sarîrat signifie effectivement à la lettre petites charrettes, mais la suite de la description de Jabarti ne laisse aucun doute, il s'agit bien de brouettes.

${ }^{7}$ Le texte arabe de Jabarti donne bi-yadihi, littéralement : « avec ses mains ».
} 
d’animaux. Le terme arabe employé par Jabarti pour désigner une brouette est révélateur de la nouveauté et de la rareté de ce véhicule : 'araba n’est pas un mot arabe ${ }^{8}$; pour désigner les véhicules à roues (ce qui est rare), les auteurs arabes utilisaient le mot 'adjala. L'origine du vocable 'araba reste problématique. Comme la langue turque emploie également le mot 'araba pour le chariot à avant-train mobile et arabasé pour désigner aussi bien de petits chariots à trois roues qu'une brouette à une roue, certains ont pensé que le mot 'araba venait du turc, ce qui est contesté par les turcologues'. Il est donc encore impossible de répondre à cette question d'étymologie.

En Iran, l'introduction de la brouette (forghun) semble, d'après nos enquêtes, se situer au $\mathrm{XIX}^{\mathrm{e}}$ siècle, mais son utilisation à une plus grande échelle ne remonte qu’à une trentaine d'années, à savoir les années 1970 (Bulliet I990 : 222). Comme en Égypte, et dans le monde arabo-musulman (avec, peut-être, une petite différence dans le cas du Maghreb du fait de la colonisation française), le transport a continué à s'effectuer selon les modes traditionnels du portage humain et d'animaux de bât. Nos enquêtes de terrain en Iran nous ont d'ailleurs permis d'apprendre que, lors des fêtes royales de Persépolis en 197I, la brouette n’était presque pas employée. Le transport de la terre et autres petits matériaux se faisait avec des sortes de paniers, de couffins, ou sacs de peaux, appelés en turc zanbil. On se servait également d'un bidon de pétrole coupé en deux, auquel avait été fixés deux brancards, tenus par deux ouvriers, un devant, un derrière, exactement comme dans les miniatures persanes du $\mathrm{XV}^{\mathrm{e}}$ siècle, sauf quà cette époque, il s'agit d'une planche de bois avec deux brancards (zanbeh).

Aujourd'hui, la brouette est présente un peu partout en Iran, notamment sur les chantiers de construction. Il est toutefois remarquable que lorsque lon pose la question du nom pour la désigner, on obtient de nombreuses réponses divergentes. Ce flou n'est pas un hasard, il est lindice de lintroduction récente de l'instrument. En Iran, la brouette est désignée, selon linterlocuteur, par les mots : forghun (du mot français fourgon ?), ou carx-é-dasti (roue à main), voire carx-é-bârbari (roue de transport de charge).

\footnotetext{
${ }^{8}$ Je remercie Pierre Larcher (Université de Bordeaux) pour les informations qu'il m'a communiquées; voir également L'Encyclopédie de l'Islam (1991 : articles : 'araba et 'adjala).

${ }^{9}$ Entretien privé avec Louis Bazin (2003).
} 


\section{Le diable}

En France (et en Europe probablement), le diable est mentionné malheureusement sans illustration - à partir du XVIII ${ }^{\mathrm{e}}$ siècle. On trouve la définition suivante dans L'Encyclopédie de Diderot et d'Alembert: «Diable: [...] se dit d'une machine à deux roues dont se servent les charpentiers pour porter quelques morceaux de bois". Et voici celle que donne le dictionnaire Littré de 1874 : «Diable (20): machine à 2 ou 4 roues pour le transport de caisses d'orangers ou autres fardeaux" et (sens 2I): " espèce de calèche dans laquelle on peut se tenir debout. "

Il est done fort probable qu'il faille faire remonter l'existence du diable au minimum à quelques décennies avant (si ce n'est quelques siècles ?), c'està-dire au début XVIII ${ }^{\mathrm{e}}$, voire au XVII ${ }^{\mathrm{e}}$ siècle. Le diable habituel (fig. 3 ) se caractérise par une grande maniabilité et un transport en plan incliné d'objets et de matériaux difficilement transportable avec une brouette: planches d'un certain format, vitraux, vitres, etc. Il permet le transport, par un seul homme, de charges importantes (en moyenne de $200 \mathrm{~kg}$ ). Pratique, mobile, individuel, comme la brouette, il permet également de se déplacer sur des voies et des passages étroits ainsi que dans les ruelles des villes pour transporter des matériaux plats et larges. De nos jours, on le retrouve dans à peu près tous les endroits du monde où se pratiquent des activités commerciales et de construction.

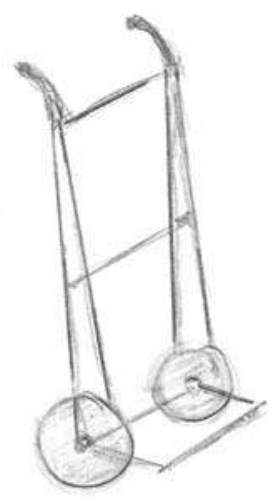

Figure 3. Diable. 
Quant à l'utilité du diable, nous pouvons aisément paraphraser ce qu'écrivait à juste titre B. Gille à propos de la brouette : quil ne faut pas dire quill s'agit là d’un instrument secondaire. Il a largement facilité, et très largement, les activités commerciales et les grands travaux d'utilité publique. Bien plus, il est, jusqu’à nos jours, beaucoup plus que d'autres, un instrument indispensable, que l'on voit dans de très nombreux secteurs économiques et commerciaux, que l'on rencontre dans toutes les zones industrielles et commerciales du monde entier, comme dans les bazars et souks du Moyen-Orient.

L'utilité et l'efficacité du diable pour tout ce qui concerne les transports de charge dans tous les entrepôts, chantiers et magasins du monde contemporain, sont indiscutables.

Nous avons vu que le diable est attesté en Europe depuis l'époque moderne et peut-être dès le bas Moyen Âge. D’après un certain nombre d'indications, il était utilisé par les charpentiers et les maçons, mais il faut admettre que les informations sont rares et que l'on reste à plusieurs niveaux dans lincertitude. Tout d'abord sur le plan linguistique et philologique : il nous a été jusqu’à présent impossible de comprendre pourquoi ce petit véhicule à roue a été appelé un diable ${ }^{10}$. Ce fait bien curieux reste une question ouverte. D'autre part, le diable dérive-t-il de la brouette ou est-ce linverse ? Est-il une invention parallèle ? Ou encore dérive-t-il également et tout simplement, comme la brouette, de la charrette (à deux roues) ? Là encore, il est pour l’instant difficile de répondre.

\section{La diffusion du diable}

On ne rencontre pas de diable jusqu'aux $\mathrm{XIX}^{\mathrm{e}}$ et $\mathrm{XX}^{\mathrm{e}}$ siècles ailleurs qu'en Europe et c'est de là qu’il se diffusera dans le reste du monde ${ }^{11}$. Si la brouette apparaît bien pour la première fois en Chine, le diable n'y est connu qu’avec la présence européenne. Il en est de même pour ce qui concerne la civilisation islamique. En Iran, le diable est désigné, selon linterlocuteur, par les mots carx-é dasti ("roue à main") ou carx-é bârbari ("roue de transport de charge»); ce flou dans le vocabulaire est également le signe

\footnotetext{
${ }^{10}$ Il a existé une charrette à deux grandes roues possédant un très long plateau central qui servait au transport de longs morceaux de bois, appelée « diable » pour une raison inconnue -peut-être parce qu'on y transportait les morts lors des épidémies?

11 Notamment aux États-Unis.
} 
d’un emploi récent, plus récent encore que pour la brouette, ce qui nous a été confirmé par nos interlocuteurs au cours de nos diverses enquêtes et qui d'ailleurs se constate encore par le faible nombre de diables, au regard des gâri-yé dasti (chariot à main), que l'on trouve dans les bazars iraniens. Mais en dix ans d'observation (1993-2003), il est incontestable que le nombre de diables a augmenté, au point de se substituer progressivement au gâri-yé dasti traditionnel. Le diable sert à de nombreux transports de matériaux de lintérieur des bazars jusqu'aux rues extérieures où ils sont transbordés dans des vanettes (petites camionnettes de fabrication japonaise ou coréenne) ou sur des motocyclettes. En Iran, en Égypte et dans le reste du Moyen-Orient, le diable, introduit par les colonisateurs anglais et français, a une histoire proche de celle de la brouette et finalement peu différente d'un point de vue technologique.

\section{Problèmes d'invention et de diffusion}

\section{Brouette chinoise et brouette européenne : diffusion ou invention parallèle?}

J. Needham, J. Gernet et B. Gille se sont demandé, comme d'autres après eux, si la brouette européenne venait, par diffusion, de la Chine. En effet, les influences sino-mongoles en Eurasie ne manquèrent pas entre les $\mathrm{XIII}^{\mathrm{e}}$ et $\mathrm{XIV}^{\mathrm{e}}$ siècles. Si diffusion il y eut, la voie italienne est la plus probable, car parmi les Européens, ce sont surtout les Italiens qui sont présents dans l'empire mongol dès le XIII ${ }^{\mathrm{e}}$ siècle (Gazagnadou 1994). Il y a en effet des colonies italiennes installées sur les bords de la mer Noire et des marchands et voyageurs italiens chez les Mongols de la Horde d'Or et dans l'Ilkhânat des Mongols d'Iran. L'armée mongole qui s'empare de Badgad en ${ }_{2} 25^{8}$ comporte des militaires et ingénieurs chinois (Gernet 2000:330); il est évident que ces derniers connaissaient la brouette, comme c'est le cas dans la tradition chinoise dès le $\mathrm{III}^{\mathrm{e}}$ siècle ap. J.-C., époque où la brouette est pour la première fois mentionnée comme ayant été utilisée par le général Shu pour ravitailler ses armées (Gernet 2000: I28; Needham I974 : 82-83). La brouette fut-elle utilisée par les armées mongoles? Nul ne le sait jusqu’à présent, mais si elle le fut, il ny a donc aucune raison pour que la brouette nait pu atteindre le Khanat mongol de la Horde d'or (mer Noire) avec 
lequel les Italiens étaient très liés. C'est ainsi que la brouette -directement et concrètement, ou lidée de la brouette, comme pour d'autres techniques (Gazagnadou 200I a et b) - a pu se diffuser et atteindre l'Europe et l'Italie en particulier. Il est à l'heure actuelle difficile de répondre tant que de nouvelles preuves ne seront pas établies.

Comment comprendre l'absence de la brouette ${ }^{12}$ au Moyen-Orient avant le $\mathrm{XX}^{\mathrm{e}}$ siècle, dans la mesure où ce n'est pas un objet technique des plus complexes ? Richard Bulliet rapporte cela au privilège, déjà mentionné, donné au transport par animaux de bât (mulets, ânes, chevaux et non seulement au chameau comme l'affirme cet auteur) ou au portage humain, par rapport aux véhicules à roues (char, chariot, charrette, et donc brouette). Pour Richard Bulliet, ce privilège serait essentiellement lié aux avantages économiques du transport animalier et par voie de conséquences, au développement d'une "mentalité" de sociétés sans roue. Nous avons montré de notre côté que les petits chariots à main à deux trains non mobiles (gari-yé dasti) utilisés par les porteurs (hammal) de marchandises des bazars iraniens étaient le résultat d'une tradition technique qui «s'est détournée" des moyens de transport à roues du fait de facteurs plus politiques qu'économiques. Est-il sûr que, dans une stricte logique technologique, on puisse rapporter cette non-diffusion ou non-invention de la brouette, en Iran ou en Égypte, uniquement au fait que les sociétés moyen-orientales ont peu employé les chariots, les charrettes, et les véhicules à roues en général ? La réponse ne nous semble pas si simple. En effet, la fabrication de chars, charrettes et chariots exige un assez grand savoir technique, leur emploi nécessite des conditions extérieures assez contraignantes, comme un certain type de géographie, des routes, donc des travaux publics, donc un appareil d'État relativement territorialisé et centralisé. Mais, ces conditions ne sont pas requises dans le cas d'une brouette ou d’un diable, véhicules assez simples liés à un seul homme et à des aires de circulation limitées. Ce cas de non-diffusion entre le monde chinois et le Moyen-Orient dans les périodes prémodernes reste ainsi non résolu.

En revanche, la diffusion très tardive de la brouette et du diable confirme une fois encore le fait que lactualisation de la tendance technique

${ }^{12}$ Cette question se pose de même pour le diable. 
(concept cher à André Leroi-Gourhan ${ }^{13}$ ) est irréversible. Il ne sagit que d'une question de temps, elle s'actualise directement dans linvention ou plus tardivement, par diffusion plus ou moins rapide. Cette diffusion s'effectue par des réseaux plus ou moins complexes et selon des modalités spécifiques, du fait de la hiérarchie entre les différentes techniques, instruments et objets techniques. C'est le contexte politique et la plus ou moins grande complexité de la technique qui expliquent tant la capacité de cette dernière à se diffuser (à se décontextualiser) qu'à ne pas se diffuser, et à ne pas être empruntée par des groupes ethniques et techniques, voisins d'un riche milieu technique.

\section{D'un système technique à l'autre}

Le système technique de transport iranien, proche et moyen-oriental était, pour l'essentiel, fondé depuis l'Antiquité sur l'articulation entre transport par animal de bât et transport à dos d'hommes, voire parfois par bateau et barque. Le transport animalier (mules, ânes, dromadaires, chameaux, chevaux) et le transport par portage humain furent les modes et les techniques de transport privilégiés dans l'Iran prémoderne et la civilisation islamique durant presque quinze siècles.

Ce système de transport aura duré plus longtemps si l'on prend en compte le système technique préislamique, qui devait avoir de nombreux points communs avec celui qui lui succéda du fait de la continuité des systèmes techniques. Et, en cinquante ans à peine, il aura été supplanté par un tout nouveau système de transport, d'origine européenne, diffusé à l'échelle mondiale et évidemment lié au système industriel moderne. Ce passage fulgurant à un système technique mécanisé, mettant fin à des pratiques si anciennes, interroge l'anthropologie des techniques du point de vue des faits proprement techniques, mais aussi sur les plans cognitif, psychologique et culturel. En effet, la longue durée du système traditionnel suggère, dans un premier temps, lỉée d'une rigidité des cadres technoculturels, qui conduirait au non-emprunt. Or, on constate que rien n'a empêché le passage -et même, peut-être a-t-il été favorisé en raison du

\footnotetext{
13 «La tendance [technique] a un caractère inévitable, prévisible, rectiligne; elle pousse le silex tenu à la main à se munir d'un manche, le ballot traîné sur deux perches à se munir de roues. » (Leroi-Gourhan 1971: 27).
} 
différentiel important d'efficacité- à un tout autre système technique, qui bouleverse non seulement les techniques, mais également les cadres sociaux et mentaux de toute une société et de toute une civilisation, en particulier par le développement considérable de la mobilité. Cela confirme l'étonnante plasticité du système cognitif humain (plasticité intellectuelle, psychique, affective, perceptive), présente sous l'apparence de la rigidité -dans le domaine de la technique comme dans d'autres. C'est pourquoi Marc Bloch (1983: 827) peut, à juste titre, écrire : "Qu'on ne s’y trompe point cependant. Emprunts et inventions portent, à y bien regarder, le même témoignage : celui d'une remarquable souplesse de la main, du regard et de l'esprit ». Cela permet de penser que dans ces sociétés en pleine transformation, quelles que soient les énormes difficultés auxquelles elles sont confrontées, les possibilités de changements radicaux sont importantes. Et du même coup, si prompts que nous soyons à juger des réels exploits technologiques et scientifiques réalisés par les sociétés industrielles, de telles capacités de mutations portent à la modestie et devraient nous conduire à chercher, à comprendre, voire, quand cela est possible, à apporter une contribution à l'amélioration des transferts de techniques à venir.

D. G.

\section{Références}

Bloch, Marc I983 "Les “inventions” médiévales ", Mélanges Historiques, tome 2. Paris : Éditions Serge Fleury -EHESS.

Bulliet, Richard W.

1990 The Camel and the Wheel. New York-Oxford : Columbia University Press.

Camarasa, Marquis de 1925 Causeries brouettiques. Madrid-Paris : libr. Berger-Levrault.

Collectif 
I99I Encyclopédie de l'Islam. Leyden : Éditions E-J. Brill (zème édition).

Cresswell, Robert

I996 Prométhée ou Pandore. Propos de technologie culturelle. Paris : Éditions Kimé.

Diderot, Denis \& Jean D'Alembert

I751 L'Encyclopédie. Tome ier. Paris : Le Breton \& Altri.

Gazagnadou, Didier

1994 La Poste à relais : la diffusion dune technique de pouvoir à travers l'Eurasie, Chine-Islam-Europe. Paris : Éditions Kimé.

1999 "Le chariot à main iranien (gâri-yé dasti): modes de transport, rationalité technique et logique d'État ", Techniques \& culture 33 : I45-I65.

200Ia «Les étriers : contribution à l'étude de leur diffusion de l'Asie vers les mondes iranien et arabe ", Techniques \& culture 37 : I55-171.

200Ib «Un savant chinois en terre d'Islam », in Floréal Sanagustin (ed.), L'Orient au coeur, Mélanges en l'Honneur d'André Miquel. Paris : Éditions Maisonneuve et Larose : 223-228.

Gernet, Jacques

2000 Le Monde chinois. Paris : Éditions Armand Colin.

Gille, Bertrand

I983 "Petites questions et grands problèmes : la brouette ", in $\mathrm{La}$

Recherche en histoire des sciences. Paris : Éditions du Seuil

(«Points sciences») : 79-88.

Jabarti, 'Abd al-Rahman

I965 'Ajaỉb al-athar wa tarâjim al-akhbâr. Le Caire, vol. 4.

I979 Journal d'un notable du Caire durant l'expédition française (I798I8OI). Traduit et annoté par Joseph Cuoq. Paris : Éditions Albin Michel.

Leroi-Gourhan, André

I97I L'Homme et la matière. Paris : Éditions Albin Michel ( ${ }^{\mathrm{e} \text { è }}$ édition 1943).

Lewis, M.J.T

I994 "The origins of wheelbarrow ", Technology and Culture 35, 3:453475

Needham, Joseph

I965 Science and Civilisation in China, vol. 4, part II, section 27. Cambridge : Cambridge University Press.

I974 La Science chinoise et l'Occident. Paris : Éditions du Seuil ( Points sciences "). 